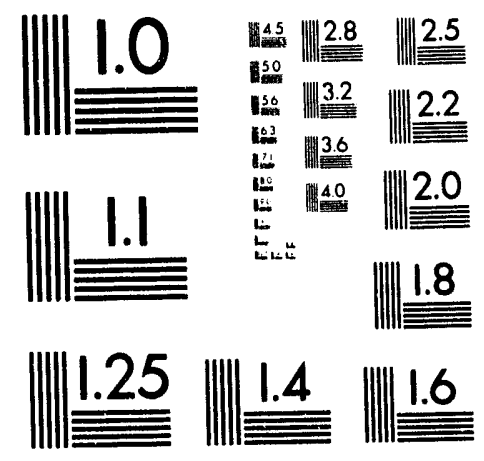



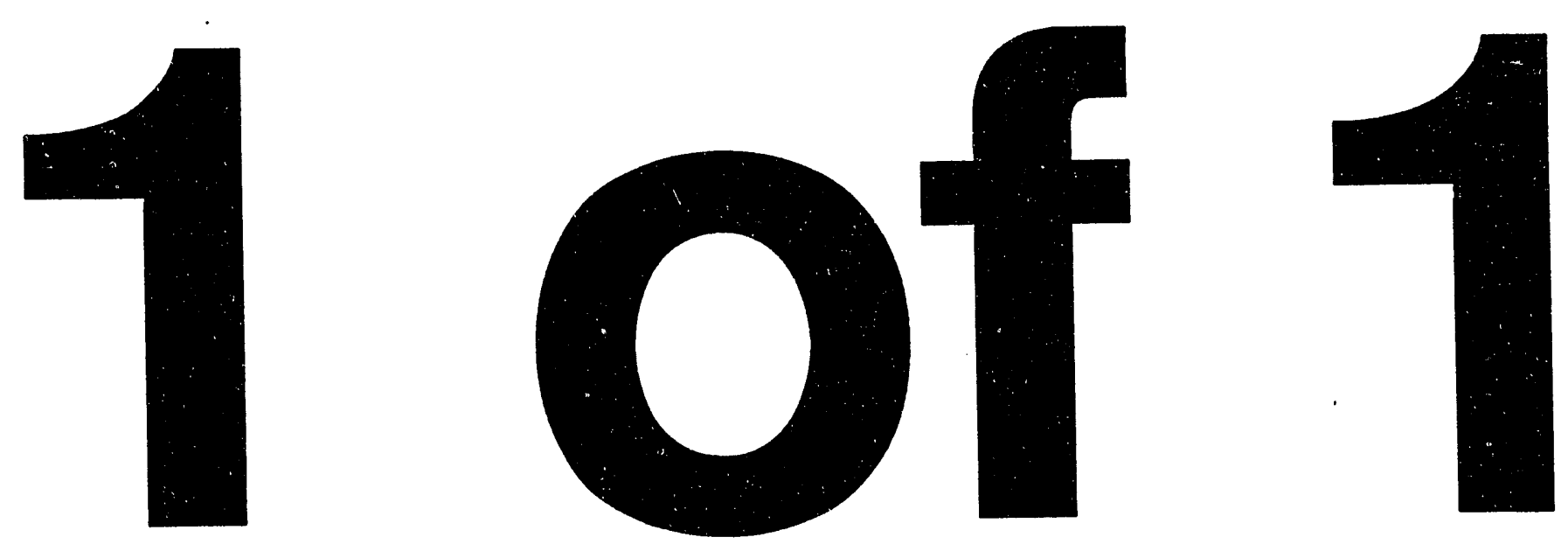
UCRL-JC-111938

PREPRINT

\title{
Computational Model of a Magnetic Modulator for Copper Lasers
}

\author{
C. D. Boley
}

This paper was prepared for submittal to the Ninth IEEE Pulsed Power Conference to be held in Albuquerque, New Mexico on June 21-23, 1993

April 1993

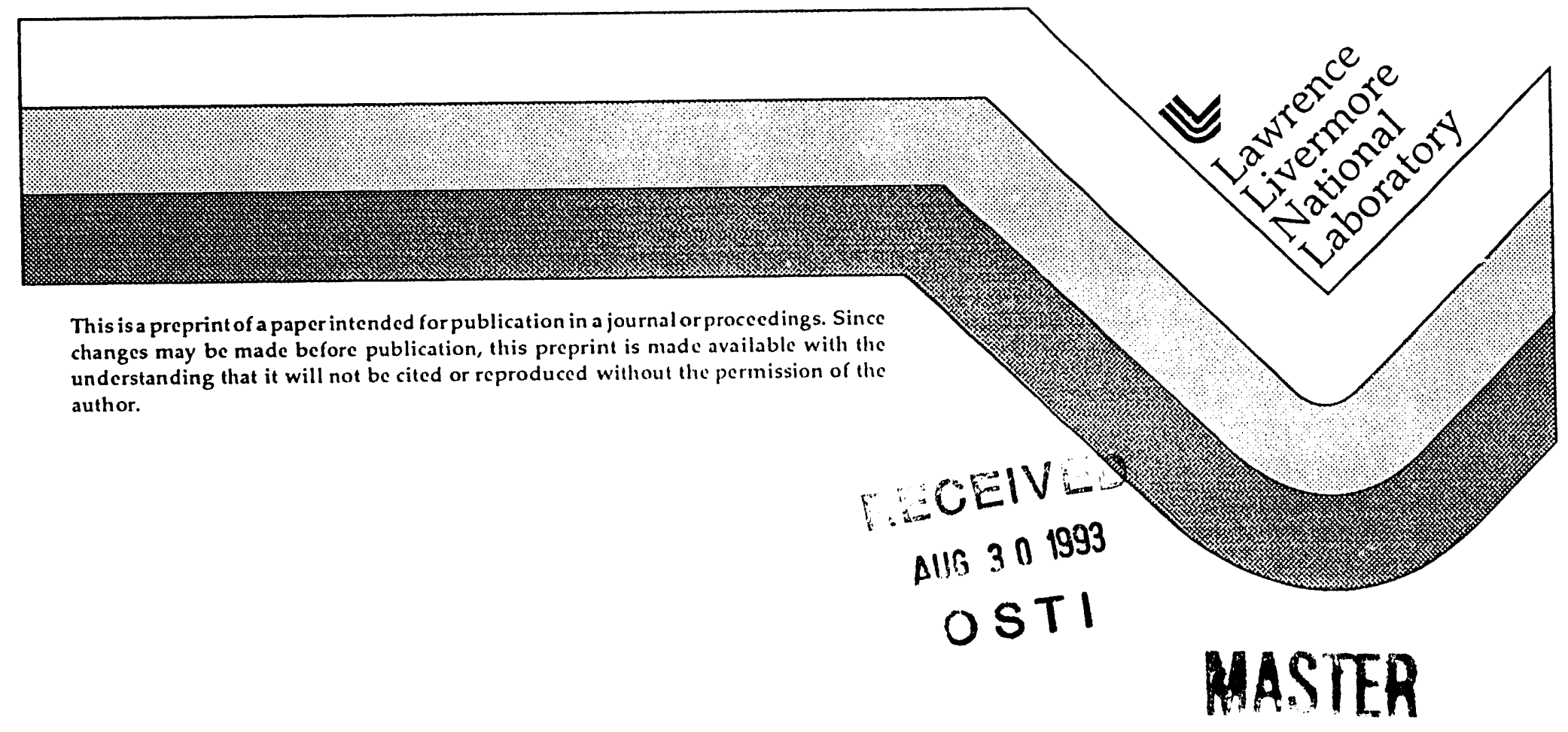




\section{DISCLAIMER}

This document was prepared as an account of work sponsored by an agency of the United States Government. Neither the United States Government nor the University of California nor any of their employees, makes any warranty, express or implied, or assumes any legal liability or responsibility for the accuracy, completeness, or usefulness of any information, apparatus, product, or process disclosed, or represents that its use would not infringe privately owned rights. Reference herein to any specific commercial products, process, or service by trade name, trademark, manufacturer, or otherwise, does not necessarily constitute or imply its endorsement, recommendation, or favoring by the United States Government or the University of California. The views and opinions of authors expressed herein do not necessarily state or reflect those of the United States Government or the University of California, and shall not be used for advertising or product endorsement purposes. 


\title{
COMPUTATIONAL MODEL OF A MAGNETIC MODULATOR FOR COPPER LASERS
}

\author{
C. D. Boley \\ University of California \\ Lawrence Livermore National Laboratory \\ Livermore, CA 94550 USA
}

\begin{abstract}
A numerical model of a three-stage magnetic modulator for copper lasers is described. The model follows in detail the hysteresis behavior of the magnetic switches and a transformer. The laser is treated via a simple model of field diffusion. The calculated voltage across the laser and current through the laser are shown to compare favorably with experiment.
\end{abstract}

\section{Introduction}

This paper presents simulations of a magnetic modulator which was developed to drive copper lasers used in isotope separation [1]. The circuit is shown schematically in Fig. 1; parameter values are listed in Table 1. The modulator uses a thyratron or SCR stack and three stages of magnetic compression to provide a $40-45 \mathrm{kV}$ pulse to the laser at a repetition rate of $4.5 \mathrm{kHz}$. It operates at an input power of $28-36 \mathrm{~kW}$. Each stage has a saturable inductor consisting of several cores made of the ferrite C/7D. There is also a 1:4 step-up transformer wound with Metglas 2605S3A.

Initially the capacitor $C_{0}$ is charged to a typical voltage of $18 \mathrm{kV}$. The nagnetic switches are held in negative satutation by reset currents. All other voltages and currents are zero. After the thyratron or SCR stack closes, $\mathrm{C}_{0}$ discharges upon $\mathrm{C}_{1}$ with a charge time of approximately $900 \mathrm{~ns}$. During this time the first magnetic switch holds off the current by presenting a large inductance to the circuit. It then saturates, allowing the energy to fow to $C_{2}$, which has a charge time of $350 \mathrm{~ns}$. When the second swith saturates. the energy. flows to $C_{3}$ (cliarge time of $90 \mathrm{us}$ ), and the voltage is stepped up by a factor of 4 by the transformer. After saturation of the third switch, the voltage is transferred to the peahing capacitor (rise time of $30 \mathrm{~ns}$ ) and then to the laser load. It then reflects back through the circuit, undergoing expansion in successive stages.

The reset circuit, not shown in Fig. 1, consists of a rod passing through each core, connected in series with a fixed voltage s.jurce, a large inductor, and a resistor.

The tast of the modet is to describe the detailed bethavior of the voltages, currents, and fields it the modulator system. Wie shall see that this can be done in quite reasonable accordance with experiment.

\section{Maguetic Model}

We employ a simple core model [2] in which the magnetic field within the cores, in both the switches and the transformer. is tahen to be azimuthal and to depend only on time, and the displacement current is ignored. The voltage arross each core then satisfies an equation of the form

$$
I^{\prime}(1)=l(1) \frac{d(1)}{d t}
$$

where the time dependent indurance is direclly proportional to the

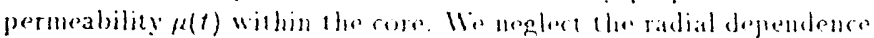
of the fields, which is an effect of onder 10\%. The magnetic field it ...lf crolves according to

$$
B(t)=\frac{1}{2 \pi} \mu(t)(t)
$$

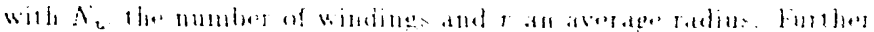

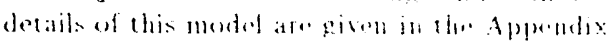

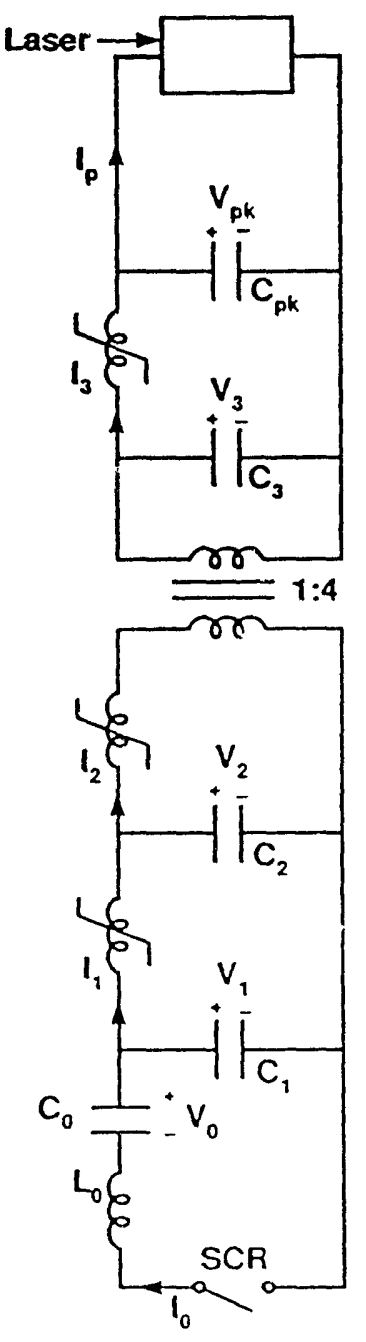

Fig. 1. Schematir modulator circuit. The reset circuit is not shown.

Table 1. Modulator parameter balues. Resistances listed next to

\begin{tabular}{|c|c|c|c|c|}
\hline & Stage 1 & Slatge? & Siage 3 & Transformer \\
\hline Material & $(: / 71)$ & $(1 / 7)$ & $(\because / 7)$ & 2605533 \\
\hline Number of couses & 10 & $\therefore$ & 10 & 1 \\
\hline Numbles of windings: & 5 & $\because$ & 2 & $f($ socollidary) \\
\hline Innes radius $\mid 1+m$ ) & $4 \therefore$ & 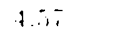 & $4 \therefore$ & 1.45 \\
\hline ()utre radius a 111 ) & 7.37 & $7 \ldots i$ & 7.37 & 7.(i) \\
\hline ('ore heightl $(1 \mathrm{~m})$ & $1.2 \%$ & $1.2 \%$ & $1.2 \%$ & 10.16 \\
\hline Salurated 1 (nilt) & 6.35 & (ii) & 450 & 11 (primary) \\
\hline Packing factor & .95 & .97 & .5 & 6.5 \\
\hline
\end{tabular}
capacitances are nominal ESR values.

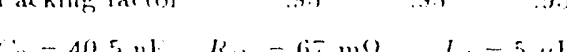

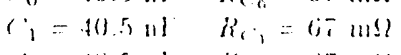

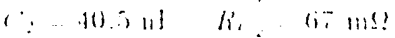

$\therefore$ inl li. : inlim!

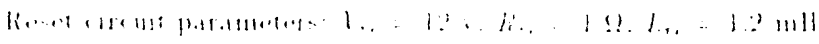

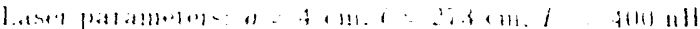


Hysteresis properties are contaned in the permeability $\mu(t)$. In the model, we employ a hysteresis theory developed by Hodgdon [3], according to which the permeability varies with $B, \dot{B}$, and $H$ in the inanner summarized in the Appendix. This theory reproduces many of the known features of hysteresis, such as loop closure near saturation, the formation of minor loops, and loop broadening with increasing $\dot{B}$. The theory requires several material parameters; those appropriate to $\mathrm{C} / 7 \mathrm{D}$ and Metglas 2605S3A are given in the Appendix.

In previous applications $[2,4]$, this combination of core model and hysteresis model was shown to agree satisfactorily with experiment.

We employ a simple model of the trigger switch (thyratron or $\mathrm{SCR}$ stack). For positive currents, it is treated as a negative $500 \mathrm{v}$ source, to account for the forward drop across the switch. For negative currents, it is modeled as a $400 \mathrm{ohm}$ resistor in series with the same source.

\section{Laser Model}

From the point of view of the modulator, the laser plays the role of a complicated load. For purposes of understanding modulator behavior, it suffices to treat the laser as a cylindrical plasma with constant resistivity $\rho$, in series with the fixed inductance $L_{c}$ of the laser container. Obviously this model of the laser cannot predict detailed properties such as the optical power.

We adopt a one-dimensional model in which the electric field $E(r, t)$ points in the $z$ direction and the magnetic field $B(r, t)$ is purely azimuthal. In the absence of displacement current, the mag. netic field then satisfies a diffusion equa ion of the form

$$
\frac{\partial B}{\partial t}=\frac{\partial}{\partial r} \frac{\rho}{\mu_{0} r} \frac{\partial(r B)}{\partial r}
$$

with a boundary value at the radius a corresponding to the plasma current: $B(a, t)=\mu_{0} I_{p}(t) / 2 \pi a$. At each instant, the electric field is given as

$$
E=\frac{\rho}{\mu_{0} r} \frac{\partial(r B)}{\partial r}
$$

and thus is not an independent dynamical quantity. The appropriate value of the resistivity, obtained by comparing a more detailed model with experiment, turns out to be approximately $.3 \mathrm{ohm} \mathrm{cm}$.

\section{Method of Solution}

It is convenient to formulate the model as a system of ordinary differential equation.: suitable for an ODE solver. The circuit equations are already in this form. although the hysteresis model presents extreme nonlinearities and jumps in the form of the equations.

To handle field diffusion in the plasma, we follow a conventional approach in which the magnetic field is assigned values at $N$ nodal points distributed evenly from the center to the edge. In practice, it was found sufficient to take $N=9$. At points 2 through $N-1$, the field then satisfies the three-point difference equation

$$
\left.\dot{B}_{1}=\frac{p}{\mu_{0} k_{1}=1} a_{1}^{+} B_{1+1}+a_{1}^{0} B_{1}+a_{1}^{-} B_{1-1}\right] \text {, }
$$

with

$$
a_{1}^{+}=\frac{i}{i-\frac{1}{2}} . \quad a_{1}^{0}=-\frac{2(i-1)^{2}}{\left(i-\frac{1}{2}\right)\left(i-\frac{3}{2}\right)} \cdot \quad a_{1}^{-}=\frac{i-2}{i-\frac{3}{2}} .
$$

and o the spacing between points. Of course $B_{1}$ is identically zero. A consistent approximation for the edge value, obtained in part by differentiating the boundary condition, is

$$
B_{X}=(1),-\left(E_{N-1}\right) /\left(2 \pi a L \cdot / \mu_{1}+\delta / / 2\right) .
$$

where the value of the electric field in the outermost cell is

$$
E_{N-\frac{1}{2}}=\frac{\rho}{\mu_{0} \delta}\left(a_{N^{\prime}-1}^{+} B_{\Lambda}-a_{N} B_{N-1}\right)
$$

Here $V_{p k}$ is the voltage across $\mathrm{C}_{p k}$ and its equivalent series resistance, and $\ell$ is the length of the laser.

The final model, therefore, consists of a system of 15 circuit variables ( 5 voltages, 6 currents, and 4 core magnetic fields), along with the $N-1=8$ magnetic field variables in the laser. Because of the core magnetic model, this is a stiff system of ODEs. We advance it in time via the solver LSODKR, a variant of the package LSODE [5], which employs Krylov methods in numerical iterations. The solver also has root-finding capabilities which are used in transitions between branches of the hysteresis curves.

\section{$\underline{\text { Results }}$}

After the voltage pulse is compressed and reflects back to $\mathrm{C}_{0}$, a recovery system stores energy for the next pulse. Since this system is not treated by the model, simulations are stopped when the voltage across $\mathrm{C}_{0}$ reaches a minimum. This occurs about $2.6 \mu \mathrm{s}$ after the thyratron/SCR is fired.

Figure 2 shows calculated voltage waveforms for $C_{0} . C_{1}$, and $C_{2}$ during this interval. As one would expect, the $900 \mathrm{~ns}$ falloff in voltage across $C_{0}$ is matched very closely by the rise across $C_{1}$. After the first magnetic switch fires, the fall across $C_{1}(350 \mathrm{~ns})$ is matched by the rise across $C_{2}$. After the second switch fires, the voltage is transferred from $\mathrm{C}_{2}$ to $\mathrm{C}_{3}$ in $90 \mathrm{~ns}$. Note that, after compression, the waveforms expand as the energy reflects back to $C_{0}$. These features are in excellent agreement with expectations based on the design of the compression system.

The calculated hysteresis behavior of the three magnetic switches is shown in Figures 3. 4, and 5. Since successive stages experience increasingly higher field frequencies, the $\mathrm{B}-\mathrm{H}$ loops broaden in width and develop minor loops. The widths, as measured at two-thirds the saturation value of $B$, are $160 \mathrm{At} / \mathrm{m}$. $260 \mathrm{At} / \mathrm{m}$. and $520 \mathrm{At} / \mathrm{m} \mathrm{in}$ the respective stages. All three stages saturate twice in the positive sense. In stage l the second saturation occurs outside the plotted domain, but in the other two stages the loop corresponding to the second saturation is visible. The dissipated hysteresis powers are $175 \mathrm{~W}, 270 \mathrm{~W}$, and $710 \mathrm{~W}$, respectively.

The voltage across the laser is depicted in Fig. 6. Note that it peaks at $37 \mathrm{kV}$. drops within $90 \mathrm{~ns}$ to a minimum of $-37 \mathrm{kV}$, and then slowly rings down with a period of about 240 ns. This behavior agrees quite weil with the measured voltage trace [G]. exrept that

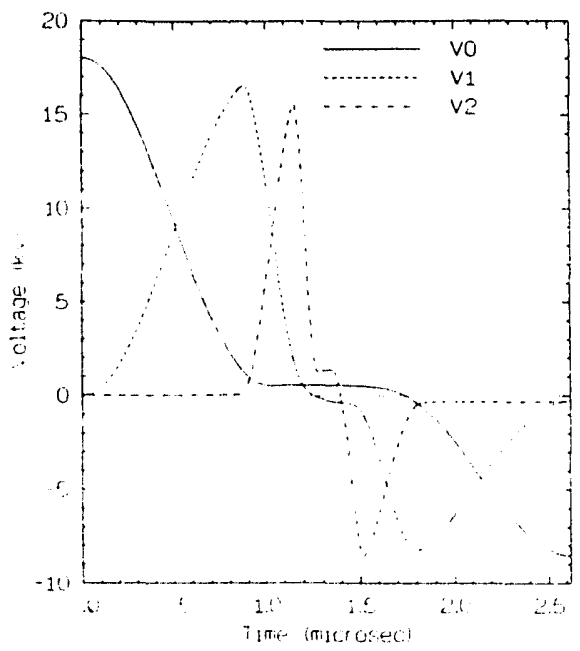

Fig. 2. Voltages across $C_{00}, C_{1}$, and $C_{2}$. 
the latter shows somewhat less dissipation. The measurement also reveals an initial leakage, which could be modeled, if desired, by placing a capacitor across the third stage switch.

Figure 7 shows the current through the laser. The calculated current peaks at $37 \mathrm{kA}$ and then rings down. The measured waveform reaches a maximum of about $60 \%$ of the calculated value, but there may be a substantial experimental error in this region. Model and experiment are reasonably close in the subsequent decay.

Finally, Figure 8 shows the calculated electric field in the laser as a function of time, at three radial locations. The field is largest near the edge, reaching a maximum of $38 \mathrm{v} / \mathrm{cm}$, then decays as it diffuses inward.

\section{Conclusions}

These simulations show that the modulator system can be modeled with quite reasonable accuracy. The code has served as a useful guide in understanding circuit behavior and in the design of possible modifications to the device.

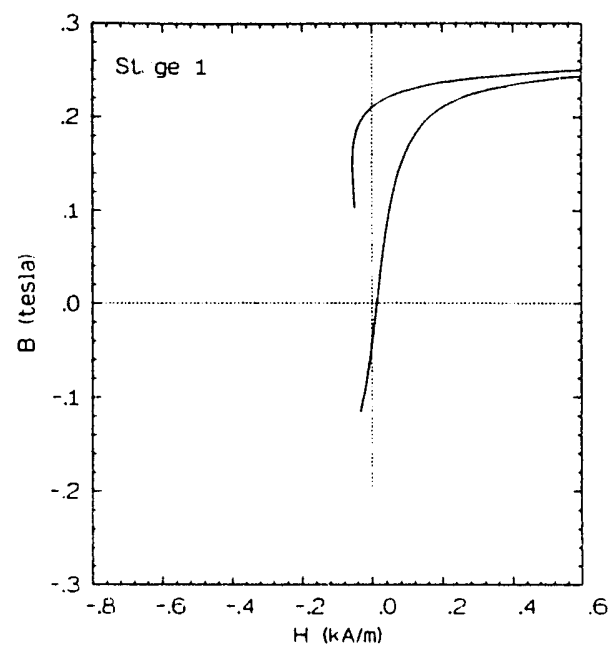

Fig. 3. Hysteresis loop of first magnetic switch.

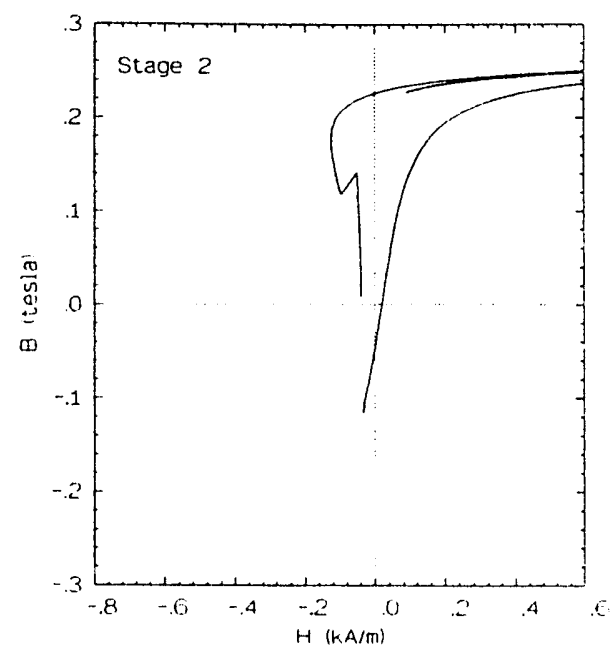

Fig. 4. Hysteresis loop of second magnetic switch.

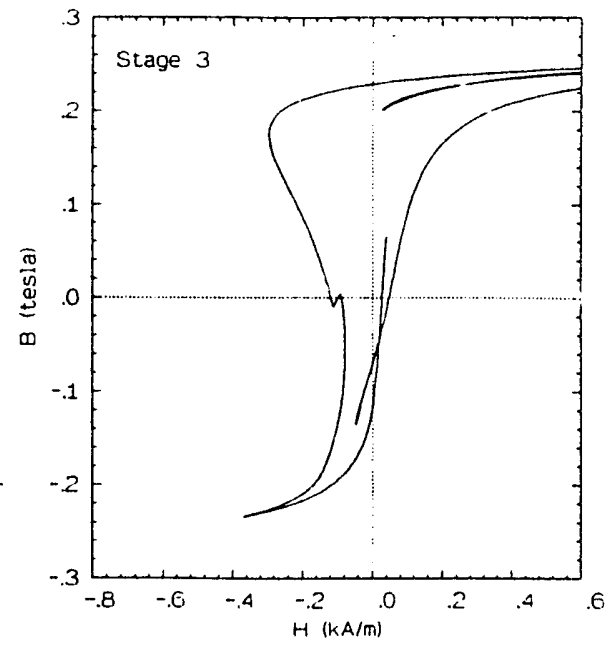

Fig. 5. Hysteresis loop of third magnetic switch.

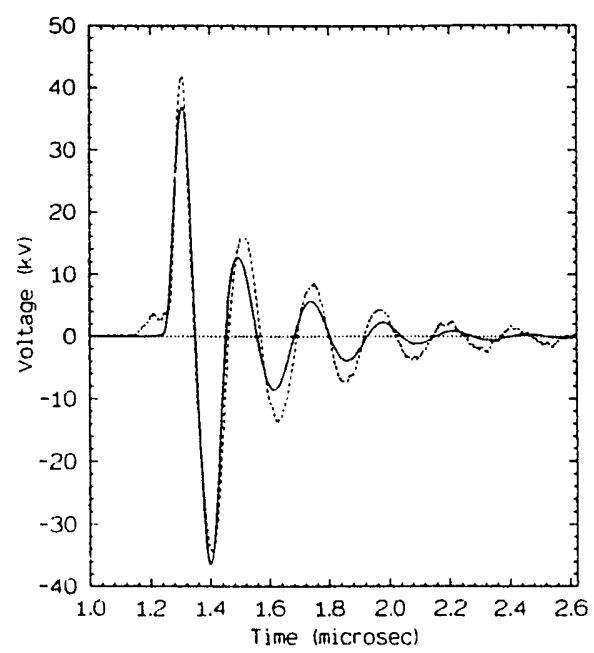

Fig. 6. Voltage across laser. The dotted line indicates Chang's data.

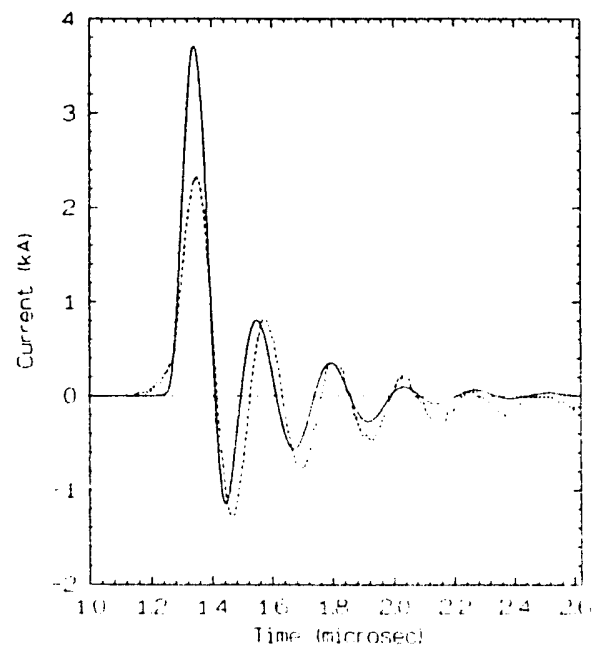

Fig. 7. Laser current. The dotted line indicates Chang's data. 


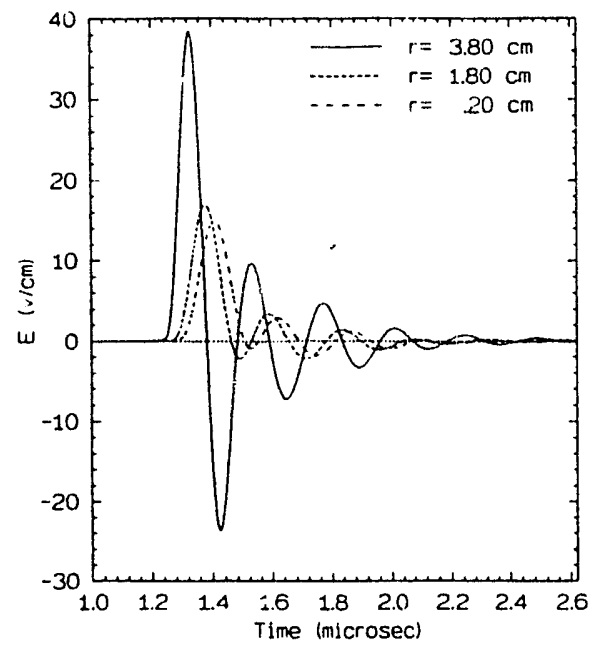

Fig. 8. Electric field in laser, at three radii.

\section{Acknowledgments}

The author is indebted to D. G. Ball, J. J. Chang, E. G. Cook, W. A. Molander, and B. E. Warner for discussions.

This work was performed under the auspices of the U.S. Department of Energy by Lawrence Livermore National Laboratory under contract No. W-7405-Eng-48.

\section{Appendix: Details of Core Magnetic Model}

Neglecting the radial dependence of the fields, the time-dependent inductance of a core is [2]

$$
L(t) \approx \frac{N_{u}^{2} h}{2 \pi} \ln \left(\frac{r_{\text {out }}}{r_{1 n}}\right) \mu(t)
$$

where $h$ is the height of a core. $N_{u}$ is the number of windings, and $r_{i n}$ and $r_{\text {out }}$ are the inner radius and outer radius, respectively. The time derivative of the magnetic field, Eq. (2). is obtained by combining the constitutive relation $\delta B=\mu \delta H$ with Ampere's law. The appropriate average radius is $\bar{r}=\left(r_{\text {out }}-r_{i n}\right) / \ln \left(r_{\text {out }} / r_{\text {in }}\right)$.

For convenience, we collect here the needed elements of the Hodgdon theory of hysteresis [3]. The permeability varies with $B, \dot{B}$, and $H$ according to

$$
\mu(B, H, \dot{B})=\{\alpha \operatorname{sign}(\dot{B})[f(B)-H]+g(B, \dot{B})\}^{-1} .
$$

where $\alpha$ is a constant and $\int$ and $g$ are functions particular to the magnetic material. The dependence of the permeability on the sign of $\dot{B}$ leads to hysteresis. The material functions $f$ and $y$ have the form

$$
\begin{aligned}
& f(B)= \begin{cases}A_{1}\left[\tan \left(A_{2} B\right)\right]^{p}+f_{c x} B, & 0 \leq B \leq B_{b} ; \\
A_{1}\left[\tan \left(A_{2} B_{b}\right)\right]^{p}+f_{c x} B_{b}+\left(B-B_{b}\right) / \mu_{s}, & B>B_{b} ; \\
-f(-B), & B<0 ;\end{cases} \\
& g(B, \dot{B})= \begin{cases}f^{\prime}(B)\left[1-A_{3} c(\dot{B}) \exp \left(-\frac{A_{4}|B|}{B_{c l}-|B|}\right)\right], & \text { if }|B|<B_{c l} ; \\
f^{\prime}(B), & \text { otherwise. }\end{cases}
\end{aligned}
$$

The rate dependence is governed by $c(\dot{B})$, which is given by

$$
c(\dot{B})= \begin{cases}1+c_{1}|\dot{B}|, & |\dot{B}|<\dot{B}_{1} ; \\ 1+c_{1} \dot{B}_{1}+c_{2}\left(|\dot{B}|-\dot{B}_{1}\right), & \dot{B}_{1} \leq|\dot{B}| \leq \dot{B}_{2} ; \\ 1+c_{1} \dot{B}_{1}+c_{2}\left(\dot{B}_{2}-\dot{B}_{1}\right)+c_{3}\left(|\dot{B}|-\dot{B}_{2}\right), & \text { otherwise. }\end{cases}
$$

The values of the constants for $\mathrm{C} / 7 \mathrm{D}$ are (mks units):

$$
\begin{array}{llll}
B_{c l}=.25 & A_{1}=39.36 & c_{1}=1.1 \times 10^{-6} & \dot{B}_{1}=4.2 \times 10^{6} \\
B_{b}=.2596 & A_{2}=5.984 & c_{2}=4.8 \times 10^{-6} & \dot{B}_{2}=\infty \\
f_{e x}=0 & A_{3}=-.5579 & c_{3}=\ldots & \dot{B}_{3}=\ldots \\
p=1 & A_{4}=.01043 & \mu_{s}=\mu_{0} & a=10
\end{array}
$$

in which $c_{3}$ and $\dot{B}_{3}$ are not needed since only the first two branches of $c(\dot{B})$ are used. For Metglas $260553 \mathrm{~A}$, the constants are:

$$
\begin{array}{llll}
B_{c l}=1.4 & A_{1}=.08734 & c_{1}=4.455 \times 10^{-4} & \dot{B}_{1}=1.1 \times 10^{5} \\
B_{b}=1.45 & A_{2}=1.0778 & c_{2}=7.868 \times 10^{-5} & \dot{B}_{2}=3.3 \times 10^{6} \\
f_{e x}=.7958 & A_{3}=-8.944 & c_{3}=5.833 \times 10^{-6} & \dot{B}_{3}=8.7 \times 10^{6} \\
p=2 & A_{4}=.2770 & \mu_{s}=.9324 \mu_{0} & \alpha=10
\end{array}
$$

\section{References}

[1] E. G. Cook et al., "High Average Power Magnetic Modulator for Copper Lasers." Proc. Eighth IEEE International Pulsed Power Conference, San Diego. CA, June 1991, p. 537.

[2] C. D. Boley and M. L. Hodgdon. "Model and Simulations of Hysteresis in Magnetic (ores." IEEE Trans. Magnetics 25, 3922 (1989).

[3] M. L. Hodgdon, "Applications of a Theory of Ferromagnetic Hysteresis," IEEE Trans. Magnetics 24, 218 (1988); M. L. Hodgdon, "Mathematical Theory and ('alculations of Magnetic Hysteresis Curves," IEEE Trans. Magnetics 24, 3120 (1988).

[4] C. D. Boley, "Simulations of a Magnetir Pulse (ompressor," Proc. Eighth IEEE International Pulsed Power Conf., San Diego, CA, June 1991, p. 704.

[5] A. C. Hindmarsh, "ODEPACK, a Systematized Collection of ODE Solvers," in Scientific Computing, R. S. Stepleman et al. (eds.), North-Holland. Amsterdaun. 195:3 (Vol. I of IMA(SS Transactions on Scientific (Computing), pp. 55-6.4.

[6] J. J. Chang, private communication. 

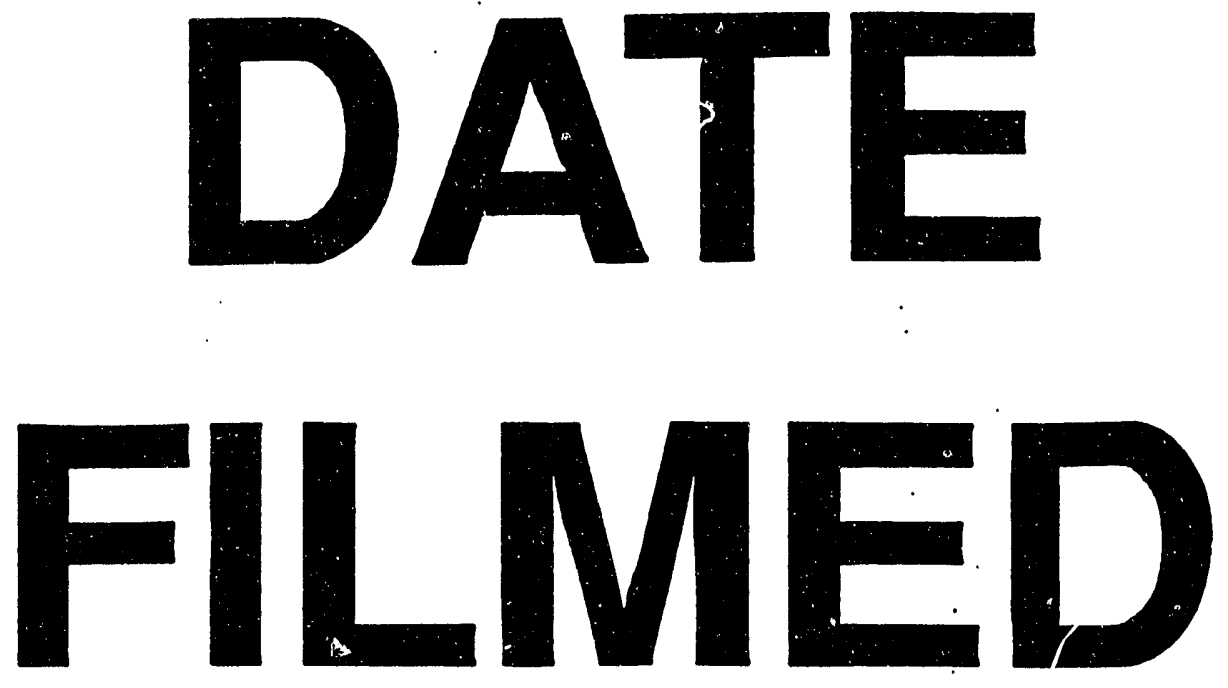

$10 / 20 / 93$
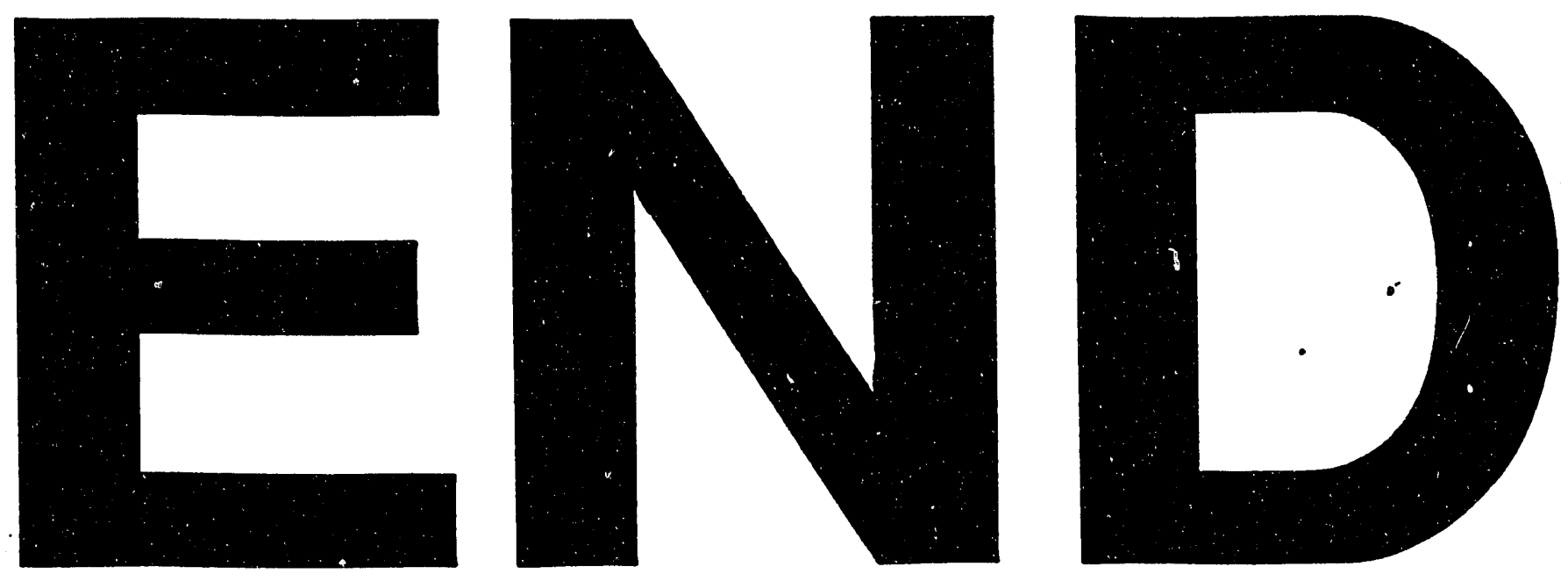
